\title{
Cerebrotendinous xanthomatosis without tendon xanthomas mimicking Marinesco-Sjoegren syndrome: a case report
}

\author{
Hartwig R Siebner, Siegmar Berndt, Bastian Conrad
}

\begin{abstract}
A 39 year old patient with cerebellar signs, juvenile cataracts, and dull normal intelligence had cerebrotendinous xanthomatosis without tendon xanthomas, diagnosed previously as Marinesco-Sjoegren syndrome. Cerebrotendinous xanthomatosis was proved by a greatly increased excretion of bile alcohols in the patient's urine. Cerebrotendinous xanthomatosis is a sterol storage disorder due to an autosomal recessive inherited defect of sterol 27-hydroxylase characterised by high cholestanol concentration in multiple tissues. If tendon xanthomas are not present, a diagnosis of cerebrotendinous xanthomatosis will often not be made, unless biochemical tests are performed. The clinical features of cerebrotendinous xanthomatosis without tendon xanthomas strongly resembles Marinesco-Sjoegren syndrome. Marinesco-Sjoegren syndrome is an autosomal recessive disorder characterised by the triad cerebellar ataxia, congenital cataract, and mental retardation. Although a late onset after the first decade of life favours cerebrotendinous xanthomatosis as the underlying disease, a definite distinction between cerebrotendinous xanthomatosis without tendon xanthomas and Marinesco-Sjoegren syndrome based on clinical presentation may be difficult. It is considered that some patients with Marinesco-Sjoegren syndrome reported in the medical literature had cerebrotendinous xanthomatosis without tendon xanthomas. This is of crucial clinical relevance, because, by contrast with Marinesco-Sjoegren syndrome, treatment for cerebrotendinous xanthomatosis is already available.
\end{abstract}

(F Neurol Neurosurg Psychiatry 1996;60:582-585)

Keywords: cerebrotendinous xanthomatosis, MarinescoSjoegren syndrome, xanthoma

Cerebrotendinous xanthomatosis is a rare autosomal recessively inherited disease characterised by tendon xanthomas, juvenile cataracts, dementia, and cerebellar and pyrami- dal abnormalities. ${ }^{1} 2$ Epilepsy, parkinsonism, and demyelinating peripheral neuropathy are other neurological features of cerebrotendinous xanthomatosis. ${ }^{1-4}$ Furthermore, psychiatric disturbances, osteoporosis, and premature arteriosclerosis with coronary artery disease have been found in patients with the disease.$^{1-4}$ More than 200 patients with cerebrotendinous xanthomatosis have been reported so far. ${ }^{14}$

Affected patients have an abnormal bile acid metabolism due to defective activity of the mitochondrial enzyme sterol 27-hydroxylase leading to abnormal cholestanol and cholesterol storage in many tissues. ${ }^{25}$ Cali et al mapped the gene to the distal portion of chromosome 2 and reported different point mutations in the gene encoding sterol 27hydroxylase, leading to a greatly diminished enzyme activity in two unrelated patients with cerebrotendinous xanthomatosis. ${ }^{5}$ Sterol 27hydroxylase is expressed in extrahepatic tissues and may play a multifaceted part in cerebral sterol metabolism that extends beyond disturbed hepatic cholesterol catabolism. ${ }^{5}$ Due to the impaired hepatic synthesis of bile acids, the patients have raised serum cholestanol concentrations and excrete large amounts of bile alcohol in bile, urine, and feces. ${ }^{1-3}$

\section{Case report}

A 39 year old man was admitted because of a slowly progressive gait disturbance. $\mathrm{He}$ was born at term after an uneventful pregnancy. Retardation in walking was noted in his first years of life. Mild mental retardation was present from infancy. Abnormality in behaviour with inattention, irritability, and agitation were noted during childhood. The patient did not finish grade school and had no further education. At nine years of age, he developed tonic seizures and was treated with phenytoin for seven years. No further epileptic seizures occurred. At 24 years of age, he underwent cataract extraction from both eyes. There was no known consanguinity in the previous generations and no other family member was clinically affected. Apart from a 42 year old brother, the patient had no living relatives. Because of juvenile cataract, ataxia, and low intelligence the diagnosis of a late onset MarinescoSjögren-syndrome was made some years ago. 
Cranial magnetic resonance imaging of the cerebellum. (A) In addition to moderate atrophy, large hyperintense areas can be seen in the cerebellar white matter on axial $T 2$

weighted turbo spin echo sequences (TR 3441, TE 120). (B) On coronal T1 weighted spin echo

sequences (TR 645, TE

16) the same areas have a decreased signal intensity.



A

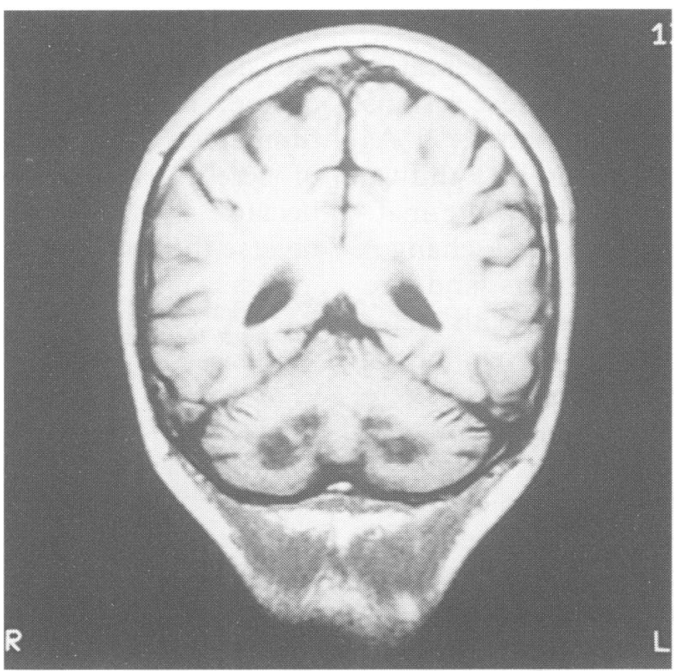

B

Physical examination showed a thin man of short stature with slight scoliosis and pes cavus deformity. His tendons were not enlarged. $\mathrm{He}$ was alert, cooperative, and oriented in all spheres. Attention and memory seemed to be moderatly impaired. The mini mental status score was 26 of a possible 30 . Evaluation of verbal and non-verbal intelligence using the multiple word choice test form B (MWT-B) and Raven's standard progressive matrices showed a low normal intelligence. Neurological examination showed a broad based atactic gait. The patient used a walking stick. Romberg's test was negative. There was mild dysmetria, decomposition of rapid successive hand movements, and pronounced finger to nose and heel to shin ataxia. Eye examination was consistent with the postoperative state of cataracts. There was horizontal nystagmus on lateral gaze.

Urinary excretion of bile alcohols

\begin{tabular}{llll}
\hline Bile alcohol component & $\begin{array}{l}\text { First urine sample } \\
\text { (mg/mol creatine) }\end{array}$ & $\begin{array}{l}\text { Second urine sample } \\
\text { (mg/mol creatine) }\end{array}$ & $\begin{array}{l}\text { Normal value } \\
\text { (mg/mol creatine) }\end{array}$ \\
\hline 23, 25-pentol & 8000 & 809 & $<5$ \\
24, 25-pentol & 1680 & 321 & $<13$ \\
27nor24, 25-pentol & Not detected & 106 & $<65$ \\
\hline
\end{tabular}

23,25-pentol $=5 \beta$-cholestane $-3 \alpha, 7 \alpha, 12 \alpha, 23,25$-pentol; 24,25 -pentol $=5 \beta$-cholestane- $3 \alpha$, $7 \alpha, 12 \alpha, 24,25$-pentol; 27nor24, 25-pentol $=27$-nor- $5 \beta$-cholestane- $3 \alpha, 7 \alpha, 12 \alpha, 22,25$ pentol. The first urine sample was collected on 31 May 1994 and the second on 7 December
Speech was slow and slurred. The other cranial nerve functions were not affected. Moderate distal muscular atrophy and paresis (4+) were present in his lower limbs. The tendon jerks of his legs were brisk, with bilateral extensor plantar responses. Touch, vibration, and joint position sense were very impaired in the feet.

Routine laboratory examinations were within normal ranges including serum cholesterol and creatine kinase. Electroencephalography was abnormal with slowed and irregular background activity. The patient showed symmetrically prolonged peripheral and central conduction times on tibial somatosensory evoked potentials and magnetic evoked potentials of the anterior tibial muscles. Latencies of early brainstem auditory evoked potentials and visual evoked potentials were delayed on both sides. Motor nerve conduction of both deep peroneal and tibial nerves were below $35 \mathrm{~m} / \mathrm{s}$. Amplitudes of compound muscle action potentials of both extensor digitorum brevis muscles were reduced. Nerve conduction velocity of the sural nerves showed a slight reduction and low distal amplitudes. Needle EMG of tibialis anterior muscles and the right biceps brachial muscle showed a chronic neurogenic pattern.

Brain CT showed bilateral symmetric cerebellar white matter hypodensities and mild cerebellar and cerebral atrophy. Magnetic resonance imaging of the brain, cervical spinal cord, and the Achilles tendons was performed by means of a superconducting magnet (1.5 Tesla), using $\mathrm{T} 1$ weighted spin echo (SE) sequences and T2 weighted turbo spin echo (TSE) sequences. Magnetic resonance imaging of the cervical spine and the Achilles tendons was normal. Cerebral MRI showed large symmetric areas of hypointensity in $\mathrm{T} 1$ weighted SE sequences and hyperintensity in T2 weighted TSE sequences in the cerebellar white matter (fig 1). In addition, there was mild cortical cerebral and cerebellar atrophy and smaller focal lesions in the pons and the paraventricular cerebral white matter. There was no enhancement after administration of gadolinium chelate.

Urinary bile alcohol excretion was measured at the Central Laboratory for Clinical Chemistry of the University Hospital Groningen, The Netherlands, using capillary gas chromatography (table). The bile alcohol content in the patient's urine was much increased proving that our patient had cerebrotendinous xanthomatosis without tendon xanthomas. His brother showed no clinical abnormality or urinary bile alcohol excretion. The patient was treated with chenodeoxycholic acid ( $250 \mathrm{mg}$ three times daily) and pravastatin (20 mg daily). After six months of treatment an improvement of gait was noted and his urinary bile alcohol content was considerably reduced (table).

\section{Discussion}

Cerebrotendinous xanthomatosis is characterised by symptoms such as dementia, ataxia, juvenile cataracts, and tendon xanthomas. ${ }^{12}$ 
However, symptoms are extremely variable in degree as well as in extent. ${ }^{36}$ In most of the patients cerebrotendinous xanthomatosis has an insidious onset in the second or third decade of life with slow progression, ${ }^{1-4}$ but an onset in the first decade of life is possible. ${ }^{7}$ The clinical hallmark of cerebrotendinous xanthomatosis are xanthomas of the Achilles tendons, ${ }^{124}$ but only a third of the patients have tendon xanthomas at onset of the disease, and $10 \%$ to $15 \%$ of patients will never develop them. ${ }^{16}$ In patients without xanthomas and without other members of the family affected, diagnosis of cerebrotendinous xanthomatosis will often not be made, even if other major manifestationssuch as bilateral cateracts, pyramidal and cerebellar deficiency, or mental abnormality-are present. ${ }^{68}$ Neurophysiological findings show diffuse but non-specific involvement of the central and peripheral nervous systems. ${ }^{9}$ Findings from CT and MRI include cerebral or cerebellar atrophy and focal or diffuse lesions of cerebral or cerebellar white matter. ${ }^{1011}$ As well as the atrophy, our patient had diffuse symmetric lesions in the cerebellar white matter on CT and MRI. However, these changes are present only in a few patients and are non-specific. ${ }^{1011}$ Because patients with cerebrotendinous xanthomatosis have raised cholestanol serum concentrations and excrete large amounts of bile alcohols in bile, urine and feces, diagnosis can be proved by measuring the urinary excretion of bile alcohols or serum cholestanol concentration. ${ }^{1-3}$ Recently, molecular genetic diagnosis of cerebrotendinous xanthomatosis has become available and provides preclinical and even prenatal diagnosis of homozygotes as well as identification of heterocygote carriers. ${ }^{12}$

Cerebrotendinous xanthomatosis can be treated with chenodeoxycholic acid. ${ }^{613}$ Due to inhibition of abnormal hepatic bile acid synthesis, chenodeoxycholic acid normalises biochemical abnormalities and has been clinically effective. ${ }^{613}$ It has not been established if a combined treatment with chenodeoxycholic acid and HMG-CoA-reductase inhibitors would be more effective than treatment with chenodeoxycholic acid alone. ${ }^{14}{ }^{15}$ Most authors emphasise that early diagnosis of cerebrotendinous xanthomatosis is mandatory to prevent irreversible damage of the nervous system..$^{1-314}$

The clinical features of cerebrotendinous xanthomatosis without tendon xanthomas strongly resemble Marinesco-Sjoegren syndrome, first described in 1931 in four Rumanian siblings. ${ }^{16}$ The basic biochemical defect of Marinesco-Sjoegren syndrome remains obscure. ${ }^{17}$ It is a rare autosomal recessive inherited disorder characterised by the triad cerebellar ataxia, congenital cataract, and mental retardation of varying degree. ${ }^{16-18}$ Additional manifestations include subnormal physical development, skeletal abnormalities, pyramidal signs, and epileptic seizures. ${ }^{16-18}$ An association with myopathy or peripheral neuropathy has been described in some patients. ${ }^{1920}$ Neuroimaging shows prominent cerebellar atrophy in patients with MarinescoSjoegren syndrome. ${ }^{17}{ }^{19}$ In most patients symp- toms were present in early infancy and the disease was either stationary or very slowly progressive during later life. ${ }^{17}$ However, several patients with Marinesco-Sjoegren syndrome of later onset and atypical clinical features have been reported. ${ }^{21} 22$ Therefore, clinical presentation may not allow a definite distinction between Marinesco-Sjoegren syndrome and cerebrotendinous xanthomatosis without tendon xanthomas. Reviewing the medical literature, it is striking that no patient with Marinesco-Sjoegren syndrome was screened for cerebrotendinous xanthomatosis. We speculate that some patients with MarinescoSjoegren syndrome reported in the past had cerebrotendinous xanthomatosis without tendon xanthomas. Cerebrotendinous xanthomatosis without tendon xanthomas has to be considered in every young patient presenting with cataracts, ataxia, or low intelligence. By contrast with Marinesco-Sjoegren syndrome, treatment for cerebrotendinous xanthomatosis is already available. Early diagnosis of cerebrotendinous xanthomatosis is crucial because medical treatment can slow or even reverse the course of the disease. ${ }^{613}$

We are indebted to Dr Wolters, Central Laboratory for Clinica Chemistry, University Hospital, Groningen, The Netherlands, for measuring the urinary bile alcohol excretion.

1 Berginer VM, Salen G, Shefer S. Cerebrotendinous xanthomatosis. In: Rosenberg RN, Prusiner S, DiMauro S, et al, eds. The molecular and genetic basis of neurological disease, London: Butterworths, 1993:295-305.

2 Björkhem I. Skrede S. Familial diseases with storage of sterols other than cholesterol: cerebrotendinous xanthomatosis and phytosterolemia. In: Scriber CR, Beaudet AL, Sly WS, Valle D, eds. The metabolic basis of inherited disease. 6th ed. New York: McGraw Hill, 1989: 1283-302.

3 Koopman BJ, Wolthers BG, van der Molen JC, van der Slik W, Waterreus RJ, van Spreeken A. Cerebrotendinous xanthomatosis: a review of biochemical findings of the patient population in the Netherlands. $f$ Inherit Metab Dis 1988;11:56-75

4 Kuriyama M, Fujiyama J, Yoshidome $\mathrm{H}$, et al. Cerebrotendinous xanthomatosis: clinical and biochemical evaluation of eight patients and review of the literature. $f$ Neurol Sci 1991;102:225-32.

5 Cali JJ, Hsieh CL, Francke U, Russell DW. Mutations in the bile acid biosynthetic enzyme sterol 27-hydroxylase underlie cerebrotendinous xanthomatosis. $f$ Biol Chem 1991;266:7779-83.

6 Waterreus RJ, Koopmann BJ, Wolthers BG, Oosterhuis HJGH. Cerebrotendinous xanthomatosis (CTX): a clinical survey of the patient population in the Netherlands. Clin Neurol Neurosurg 1987;89:169-75.

7 Wevers RA, Cruysberg JRM, Van Heijst AFJ, et al. Paediatric cerebrotendinous xanthomatosis. $\mathcal{f}$ Inherit Metab Dis 1992;15:374-6.

8 Van Hellenberg-Hubar JLM, Joosten EMG, Wevers RA. Cerebrotendinous xanthomatosis. Clin Neurol Neurosurg 1992;94(suppl):S165-7.

9 Tokimura Y, Kuriyama M, Arimura K, Fujiyama J, Osame $M$. Electrophysiological studies in cerebrotendinous xanthomatosis. F Neurol Neurosurg Psychiatry 1992;55:52-5.

10 Berginer VM, Berginer J, Korczyn AD, Tadmor R. Magnetic resonance imaging in cerebrotendinous xanthomatosis: a prospective clinical and neuroradiological thomatosis: a prospective $₹$ Neurol Sci 1994;122:102-8.

11 Hokezu Y, Kuriyama M, Kubota R, Nakagawa M, Fujiyama J, Osame $\mathbf{M}$. Cerebrotendinous xanthomatosis: cranial CT and MRI studies in eight patients. Neuroradiology 1992;34:308-12.

12 Meiner V, Meiner Z, Reshef A, Bjorkhem I, Leitersdorf E. Cerebrotendinous xanthomatosis: molecular diagnosis Cerebrotendinous xanthomatosis: molecular diagnosis Neurology 1994;44:288-90.

13 Berginer VM, Salen G, Shefer S. Long-term treatment of cerebrotendinous xanthomatosis with chenodeoxycholic acid. N Engl f Med 1984;311:1649-52.

14 Peynet J, Laurent A, DeLiege P, et al. Cerebrotendinous xanthomatosis: treatment with simvastatin, lovastatin and chenodeoxycholic acid in 3 siblings. Neurology 1991; 41:434-6.

15 Salen G, Batta AR, Tint GS, Shefer S. Comparative effects of lovastatin and chenodeoxycholic acid and abnormal bile acid metabolism in cerebrotendinous xanthomatosis. 
Metabolism 1994;43:1018-22.

16 Marinesco G, Draganesco S, Vasilia D. Nouvelle maladie familiale caractérisée par une cataracte congénitale et un arret du dévelopment somato-neuro-psychique. L'Encéphale 1931;26:97-109.

17 Mahloudi M. Marinesco-Sjögren syndrome. In: Vinken PJ, Bruyn GW, eds. Handbook of clinical neurology. Vol 21 . Amsterdam: Elsevier, 1975:555-61.

18 Alter M, Talbert R, Croffead G. Cerebellar ataxia, congenital cataracts, and retarded somatic and mental maturation. Neurology 1963;12:836-47.

19 Torbergsen T, Aasly J, Borud O, Lindal S, Mellgren SI. Mitochondrial myopathy in Marinesco-Sjögren syndrome. Fournal of Mental Deficiency Research 1991;35:
154-9.

20 Zimmer C, Gosztonyi G, Cervos-Navarro J, vonMoers A Schröder JM. Neuropathy with lysosomal changes in Marinesco-Sjögren syndrome: fine structural findings in skeletal muscle and conjunctiva. Neuropediatrics 1992;23: 329-35.

21 Monnet P, Paufique L, Salle B, Rosenberg D, Pasquier N, Picaud S. Syndrome familial du type Marinesco-Sjögren avec quelques variantes. Arch Fr Pédiatr 1969;26:87-95.

22 Nyberg-Hansen R, Grönvik O, Refsum S. Hereditary cerebellar ataxia associated with congenital cataracts. Four cases of the Marinesco-Sjögren syndrome with some unusual features. Acta Neurol Scand Suppl 1972;51: 257-60.

\section{Migraine}

Continued from this fournal 1996;60:448.

Wilfred Blunt, 1901-87, The compleat naturalist. A life of Linnaeus (1971)

On reading Malmis, Linnaeus was laid low by one of his recurrent attacks of migraine, which on this occasion resulted from drinking a glass of sloe gin Sometimes it was alcohol-sour wine in particularwhich brought them on, sometimes cold weather or strong wind. Or even a sharp disappointment. A few years later he recorded in his Autobiography an occasion when an attack was induced by annoyance at the loss, through a gardener's stupidity, of some long desired cochineal insects brought back for him by Daniel Rolander from Surinam.

He was a life long victim of migraine. We learn that prior to an attack he would often observe someone walking alongside him stopping perhaps to examine a flower. It was a little while before he realised that the man he saw was his Döppelganger... Once he entered the lecture theatre prepared to take his class, when he saw someone standing at the lectern. Thinking that he had arrived before the previous lecturer had finished, he turned away, only to realise he had been looking at a specular hallucination of himself.

\section{fohn Dryden (1631-1700)}

For all the happiness mankind can gain

Is not in pleasure, but in rest from pain.

fean-Marie Charcot (1825-93)

At one of his Tuesday clinics:

"Mon Dieu! It is not surprising that one cannot describe the shock of the scintillating scotoma. Many times I have experienced it. The first occasion when it happened I had, or thought I had, a firework display in front of me. Only later, from closer scrutiny, did I make out a sort of circle like one of Marshal Vaubain's fortification with its salients and recesses."

Winifred Holtby (1898-1935)

Constant companion of my wakeful days

And uninvited bridegroom of my bed

Withdraw a little, and thy hand upraise

From my tormented head.

Fohn Steinbeck, the wayward bus (1947)

Mrs Pritchard-" Her husband knew her headaches, and they were dreadful. They twisted her face and reduced her to a panting, sweating, grinning, quivering blob of pain. They filled a room and a house. They got into everyone around her. Mr Pritchard could feel one of her headaches through the walls... The headaches usually came when she was nervous and when things, through no fault of her own, were not going well."

Steinbeck also touched on the extra cross that the migraine victim often bears, the accusation of malingering, here by Mrs Pritchard's unfeeling daughter, who dismissed the pain as merely "psychosomatic". It was not so.

"They seemed to be selfish, these headaches, and yet they were not. The pain was real. No one could simulate such agonizing pain. Mr Pritchard dreaded them more than anything in the world. A good one could make the whole house vibrate with horror."

Alexander Pope, 1688-1744, A life by Maynard Mack (1985)

4 feet 6 inches. He was very hump-backed and deformed (Pott's disease of spine). He wore a black coat and, according to the fashion of the time, had a little sword. He was afflicted with constant headaches, sometimes so severe that he could barely see the paper he wrote upon.

George Bernard Shaw, 1856-1950

He suffered from headaches for most of his life, as illustrated by his meeting with the famous Arctic explorer, Nansen:

One afternoon after recovering from an attack, he was introduced to Nansen and asked whether he had ever discovered a headache cure. "No", said Nansen with a look of amazement. "Have you ever tried to find a cure for headache?" "No".

"Well that is a most astonishing thing," exclaimed Shaw. "You have spent your life trying to discover the North pole, which nobody on earth cares tuppence about, and you have never attempted to discover a cure for headache, which every living person is crying aloud for."

Michael Holroyd in the first volume of his biography of Shaw, The search for love, describes some of Shaw's attempts to overcome his monthly migraines:

Shaw's experiments encouraged in him the belief that vegetarian food might improve his general health -in particular remove the severe headaches that had started to attack him every month and this he attributed, not to anxiety or overwork, but to unscientific eating habits.

There must be benefits, he reasoned, in studying food a little instead of going on with the old unthinking habits infused on him in childhood. In a sense vegetarianism came easily to Shaw. A symptom of his neglect in Ireland had been the poor diet, the only food he had liked was the stoneground bread which his mother occasionally buttered for him. An ordinary vegetarian diet was not enough

He was of the opinion that his diet included an excess of protein. Until he was 70 he accumulated some poison that exploded every month or six weeks in a headache that blew it off and left him quite well after disabling him for a day. He tried every available treatment to get rid of the headaches and all quite unsuccessful. He now makes uncooked vegetables, chopped or grated, and their juices with fruit, the stable of his diet and finds it markedly better than the old high protein diet of beans, lentils and macaroni.

On the 19th May he started a new novel. He had been at work on this for about a week when he began to feel ill. The new vegetarian diet had not cured his headaches and, in the belief that he was spending too much time indoors he prescribed for himself a number of rides round London on the top of an omnibus. A few days later he discovered he had smallpox.

EMR CRITCHLEY Royal Preston Hospital PO Box 66 Sharoe Green Lane North Fulwood, Preston PR2 4HT, UK 